\title{
A novel stage-specific glycosomal nucleoside diphosphate kinase from Trypanosoma cruzi
}

\author{
María de los Milagros Cámara ${ }^{1}$, León Bouvier ${ }^{2}$, Chantal Reigada ${ }^{3}$, Fabio A. Digirolamo ${ }^{3}$, Melisa Sayé ${ }^{3}$ and \\ Claudio A. Pereira ${ }^{3}$ \\ ${ }^{1}$ Instituto de Tecnología, Universidad Argentina de la Empresa, Buenos Aires, Argentina; \\ ${ }^{2}$ Instituto de Investigaciones Biotecnologicas, Buenos Aires, Argentina \\ ${ }^{3}$ Laboratorio de Parasitología Molecular, Instituto de Investigaciones Médicas, Universidad de Buenos Aires and National Research \\ Council (CONICET), Buenos Aires, Argentina
}

\begin{abstract}
Nucleoside diphosphate kinases (NDPK) are key enzymes involved in the intracellular nucleotide maintenance in all living organisms, especially in trypanosomatids which are unable to synthesise purines de novo. Four putative NDPK isoforms were identified in the Trypanosoma cruzi Chagas, 1909 genome but only two of them were characterised so far. In this work, we studied a novel isoform from T. cruzi called TcNDPK3. This enzyme presents an atypical N-terminal extension similar to the DM10 domains. In T. cruzi, DM10 sequences targeted other NDPK isoform (TcNDPK2) to the cytoskeleton, but TcNDPK3 was localised in glycosomes despite lacking a typical peroxisomal targeting signal. In addition, TcNDPK3 was found only in the bloodstream trypomastigotes where glycolytic enzymes are very abundant. However, TcNDPK3 mRNA was also detected at lower levels in amastigotes suggesting regulation at protein and mRNA level. Finally, 33 TcNDPK3 gene orthologs were identified in the available kinetoplastid genomes. The characterisation of new glycosomal enzymes provides novel targets for drug development to use in therapies of trypanosomatid associated diseases.
\end{abstract}

Keywords: energy metabolism, trypanosomatids, Chagas disease, glycosomes, trypomastigotes

This article contains supporting information (Figs. S1, S2) online at http://folia.paru.cas.cz/suppl/2017-64-006.pdf

Nucleoside diphosphate kinases (NDPK) are enzymes involved in the intracellular nucleotide balance that catalyse the reversible transference of high-energy phosphates from a nucleoside triphosphate donor to a nucleoside diphosphate acceptor (Veron et al. 1994). In trypanosomatid parasites NDPKs are key enzymes due to their inability to synthesise purines de novo and thus they depend on NDPKs for nucleotide recycling (Berriman et al. 2005). This essential function allows considering NDPKs as potential therapeutic targets for trypanosomiasis.

Trypanosoma cruzi Chagas, 1909 has four isoforms of NDPK, two of them well characterised (TcNDPK1 and TcNDPK2) and other uncharacterised putative isoforms (TcNDPK3 and TcNDPK4). TcNDPK1 is the canonical short isoform, whereas TcNDPK2, TcNDPK3 and TcNDPK4 correspond to the 'long' isoform variants. Isoforms 2 and 3 have one DM10 domain preceding the catalytic region and variant 4 has unknown $\mathrm{N}$ - and $\mathrm{C}$-terminal extensions (Miranda et al. 2008a, 2011). NDPK activity in trypanosomatids was first described by Ulloa et al. (1995) detecting NDPK activity in some subcelular fractions including membranes. These authors also purified a soluble NDPK with similar characteristics to TcNDPK1. TcNDPK1 is a hexameric enzyme (Gomez Barroso et al. 2010) expressed mainly in trypomastigote and amastigote stages of the T. cruzi life cycle.

This isoform also showed DNAse activity (Miranda et al. 2008b), which was extensively characterised in NM23-H2, the human orthologous of TcNDPK1 (Postel 2003). As genomic DNA of T. cruzi was degraded by TcNDPK1 nuclease activity, this isoform could act at nuclear level as a component of programmed cell death machinery or DNA repair systems (Debrabant and Nakhasi 2003, Hammargren et al. 2007). In this sense, the Trypanosoma brucei gene orthologous of TcNDPK1 was localised in the parasites nucleus (Hunger-Glaser et al. 2000) and also in secreted parasite microvesicles (Geiger et al. 2010). In addition, the Leishmania spp. gene orthologous was associated to microsomal fractions and it was found to be secreted regulating macrophage infection (de Oliveira et al. 2006, Kolli et al. 2008).

In TcNDPK1 of T. cruzi has a cytosolic perinuclear distribution and, in a subpopulation of epimastigotes under 
metacyclogenesis and metacyclic trypomastigotes, was localised in non-membrane bounded granules adjacent to the nucleus (Pereira et al. 2014). TcNDPK2 was localised in cytoskeleton and flagella, detected in polymerised tubulin. Additionally, it has been demonstrated that the DM10 domain is responsible for TcNDPK2 microtubule association (Miranda et al. 2011). In this work we identified for the first time a nucleoside diphosphate kinase of T. cruzi localised in glycosome which is predominantly expressed in bloodstream trypomastigotes.

\section{MATERIALS AND METHODS}

\section{Cell cultures}

Epimastigotes of Y (DTU Trypanosoma cruzi II) were cultured at $28^{\circ} \mathrm{C}$ in plastic flasks $\left(25 \mathrm{~cm}^{2}\right)$, containing $5 \mathrm{ml}$ of Brain Heart Tryptose medium (started with $10^{6}$ cells per millilitre) supplemented with $10 \%$ fetal calf serum, $100 \mathrm{U} / \mathrm{ml}$ penicillin and $100 \mu \mathrm{g} / \mathrm{ml}$ streptomycin (Camargo 1964). The parasites were subcultured with passages each 7 days. Cells were counted using a hemocytometer.

\section{Fluorescence microscopy}

Epimastigote samples from the days 1-7 after transfection were washed twice with PBS. After letting the cells settle for $30 \mathrm{~min}$ at room temperature onto poly-L-lysine coated coverslips, parasites were fixed at room temperature for $20 \mathrm{~min}$ with $4 \%$ formaldehyde in PBS, followed by a cold methanol treatment for 5 min. Parasites were washed with PBS and incubated with polyclonal TcNDPK3 antibodies $(1: 200)$, followed by Alexa Fluor ${ }^{\mathbb{B}}$ 488-conjugated secondary antibody and slides were mounted using Vectashield with DAPI (Vector Laboratories, Burlingame, CA, USA). Cells were observed in an Olympus BX60 fluorescence microscope. Images were recorded with an Olympus XM10 camera.

\section{Western blot analysis}

Western blots were performed using total extracts of T. cruzi, fractioned by electrophoresis in polyacrylamide denaturing gels, and transferred to polyvinylidene fluoride (PVDF) membranes. The PVDF membranes were treated for $1 \mathrm{~h}$ with $5 \%$ non-fat dry milk in PBS and then incubated with the corresponding primary antibody overnight, (anti-TcNDPK3 diluted $1: 2,000$ or anti-polyHistidine 1 : 3,000; Sigma-Aldrich, St. Louis, MO, USA). Membranes were washed and incubated with the secondary antibody for $2 \mathrm{~h}$ (anti-mouse HRP 1 : 2,500, Vector Laboratories). Detection was done by chemiluminescence (Pierce Biotechnology, Rockford, IL, USA).

\section{Nucleoside diphosphate kinase activity}

NDPK activity was measured using the purified TcNDPK3 recombinant protein. This protein was produced using cells of Escherichia coli transformed with an expression plasmid containing the TcNDPK3 fused to a 6His tag (pRSET-6His::TcNDPK3). The production was induced with $0.1 \mathrm{mM}$ IPTG for $4 \mathrm{~h}$. Since the protein was obtained aggregated in inclusion bodies, first these were resuspended in $50 \mathrm{mM}$ Tris- $\mathrm{HCl}$ buffer $(\mathrm{pH} \mathrm{8),} 100 \mathrm{mM}$ $\mathrm{NaCl}$ and $6 \mathrm{M}$ urea. Then, the solution was diluted in the same buffer until reaching $1 \mathrm{M}$ urea. Finally, the protein was purified using an affinity purification resin (Ni Sepharose ${ }^{\mathrm{TM}} 6$ Fast Flow, GE Healthcare, Chicago, IL, USA). The activity was measured as previously reported (Miranda et al. 2008a). Briefly, $1.5 \mu \mathrm{g}$ of TcNDPK3 recombinant protein was added to the reaction mixture (100 mM Tris-HCl buffer, $\mathrm{pH}$ 7.5, $3 \mathrm{mM}$ phosphoenolpyruvate, $10 \mathrm{mM} \mathrm{MgCl} 2,0.3 \mathrm{mM}$ NADH, $25 \mathrm{mM} \mathrm{KCl}, 1.25 \mathrm{U} / \mathrm{ml}$ pyruvate kinase and $2.2 \mathrm{U} \mathrm{ml}$ lactate dehydrogenase) in a cuvette to give a final volume of $0.5 \mathrm{ml}$. After $5 \mathrm{~min}$ at $35^{\circ} \mathrm{C}$ the reaction was started by the addition of a small volume of ATP and dTDP to final concentrations of $2 \mathrm{mM}$ and $0.1-4 \mathrm{mM}$, respectively. The activity was calculated measuring the decrease in absorbance at $340 \mathrm{~nm}$ due to the oxidation of NADH.

\section{RNA extraction and Real time PCR assays}

Epimastigote, trypomastigote and amastigote cells were harvested by centrifugation, washed with PBS and resuspended in $1 \mathrm{ml}$ of Tri-Reagent (Sigma), mixed by inversion and $200 \mu \mathrm{l}$ of chloroform was added followed by centrifugation at 12,000 $\mathrm{g}$ at $4{ }^{\circ} \mathrm{C}$. The supernatant was transferred to a clean test tube and $500 \mathrm{ml}$ of isopropanol was added; after $10 \mathrm{~min}$ of incubation at room temperature, cells were centrifuged at $12,000 \mathrm{~g}$ for $15 \mathrm{~min}$. The pellet was washed with ethanol to $75 \%$, left to dry and resuspended in $20 \mu \mathrm{l}$ of RNase-free water. RNA concentrations were determined spectrophotometrically and purity was confirmed by gel electrophoresis; $3 \mu \mathrm{g}$ of RNA were used for retrotranscription. Before retrotranscription, samples were treated with DNase I (Sigma). TcNDPK3 mRNA expression in the major T. cruzi life cycle stages was quantified by SYBR green-based real-time PCR in a Real-Time PCR system (Thermo Fisher Scientific, Waltham, MA, USA) using default protocols. Data were normalised to $18 \mathrm{~S}$ ribosomal RNA expression. Three independent experiments were carried out, for each every three independent samples.

\section{Bioinformatics}

Sequences from the TriTryp genome projects were obtained at TriTrypDB (http://tritrypdb.org/) and GeneDB (http://www. genedb.org/). Analysis of the DNA sequence data were carried out using the software package Vector NTI v. 10.3.0 (Invitrogen, Carlsbad, CA, USA) and the online version of BLAST at the NCBI (http://www.ncbi.nlm.nih.gov/). Local or online software were used under default parameters. Phylogenetic analysis was performed using MEGA v6 (Tamura et al. 2013).

\section{RESULTS}

Cloning, overexpression and functional validation of TcNDPK3. The predicted TcNDPK3 is a 349 amino acid long protein with an isoelectric point of 5.2 and a molecular weight of about $39.3 \mathrm{kDa}$. To test if TcNDPK3 is a bona fide nucleoside diphosphate kinase, the gene was fused to a x6His tag $(2.5 \mathrm{kDa})$, cloned and expressed in Escherichia coli. The affinity purified recombinant protein showed in the SDS-PAGE (Fig. S1A,B) has a predicted molecular weight of $41.8 \mathrm{kDa}$. The functionality of the purified recombinant nucleoside diphosphate kinase was fully confirmed by standard enzymatic activity using an enzymes coupled assay by the production of ADP using dTDP and ATP as substrates (Fig. 1). The enzyme presented a Michaelis-Menten kinetics, with $\mathrm{Km}=1.1 \mathrm{mM}$ and 


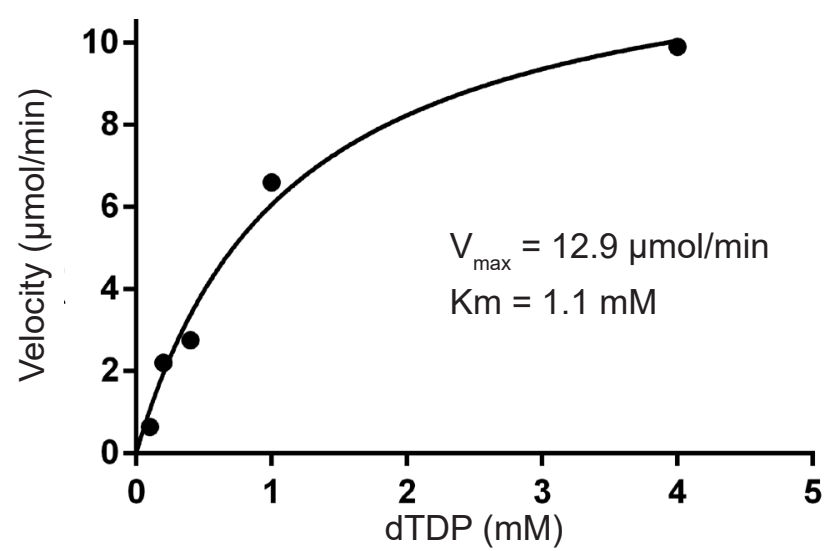

Fig. 1. Functional validation of Trypanosoma cruzi nucleoside diphosphate kinase 3 (TcNDPK3). Different dTDP concentrations (0.1-4 mM) were evaluated in order to obtain the TcNDPK3 kinetic parameters based on ADP production from dTDP and ATP as substrates. The assays were performed during $10 \mathrm{~min}$ using $1.5 \mu \mathrm{g}$ of recombinant protein.

Table 1. NDPKs kinetic parameters for dTDP as substrate from different organisms. Data obtained from BRENDA (BRaunschweig ENzyme DAtabase, www.brenda-enzymes.org).

\begin{tabular}{ll}
\hline $\begin{array}{l}\text { Km value } \\
\text { dTDP }(\mathrm{mM})\end{array}$ & Organism \\
\hline 0.03 & Acanthamoeba polyphaga Mimivirus \\
0.1 & Pisum sativum \\
0.17 & Saccharomyces cerevisiae \\
$0.21-0.39$ & Homo sapiens \\
$0.21-0.39$ & Bos taurus \\
$0.21-0.39$ & Avena sativa \\
0.42 & Plasmodium falciparum \\
0.47 & Trypanosma cruzi strain CL Brener - isozyme NDPK2 \\
0.86 & Trypanosma cruzi strain CL Brener - isozyme NDPK1 \\
1.1 & Trypanosma cruzi strain CL Brener - isozyme NDPK3 \\
\hline
\end{tabular}

$\mathrm{V}_{\max }=12.9 \mu \mathrm{mol} / \mathrm{min} . \mathrm{Km}$ values were compared with those obtained for NDPKs from other organisms besides the previously characterised isoforms of $T$. cruzi (Table 1).

Phylogenetic analysis of TcNDPKs. By data mining strategies all gene orthologs of TcNDPK1-TcNDPK4 were obtained from the available genomes from species of the genera Crithidia Léger, 1902, Endotrypanum Mesnil et Brimont, 1908, Leishmania Ross, 1903, Leptomonas Kent, 1880 and Trypanosoma Gruby, 1843. TcNDPK1-TcNDPK4 have 41, 32, 33 and 12 gene orthologs, respectively. Using these 118 genes a Maximum Likelihood phylogenetic tree was constructed (Fig. 2A). In the resulting unrooted phylogenetic tree it can be clearly seen the four clusters of the gene orthologs of the different isoforms of NDPK. TcNDPK1, the canonical isoform, grouped the higher number of genes (41), TcNDPKs possessing the N-terminal DM10 domains (TcNDPK2 and TcNDPK3; Fig. 2B) have a similar number of orthologs (32-33), and the other 'long' putative isoform of TcNDPK (TcNDPK4) has only 12 gene orthologs. Within the four isoforms of Trypanosoma cru$z i$ NDPKs, TcNDPK4 is the only one not yet functionally validated.
Expression of TcNDPK3 during the life cycle of Trypanosoma cruzi. TcNDPK3 expression was evaluated in the main stages of the life cycle of T. cruzi. Western blot analysis were performed using anti-TcNDPK3 specific antibodies and total extract samples from epimastigotes, trypomastigotes and amastigotes. TcNDPK3 signal was detected exclusively in bloodstream trypomastigote samples (Fig. 3A). To further confirm these results the abundance of TcNDPK 3 mRNA in the three stages was quantified by real time PCR (Fig. S2). The relative amounts of TcNDPK3 mRNA were calculated and expressed as arbitrary units (AU). Epimastigotes samples have 0.9 AU, whereas trypomastigotes and amastigotes samples have 10.9 AU and 6.1 AU, respectively. These results indicate that TcNDPK 3 has a stage-specific regulation and its expression is partially modulated at mRNA level.

Subcellular localisation of TcNDPK3. Among the nucleoside diphosphate kinase family of T. cruzi, TcNDPK2 is the only isoform that possesses a functionally characterised N-terminal DM10 domain which is similar to the one present in TcNDPK 3 with about $50 \%$ of consensus positions (Fig. 2B). This data suggests that, like TcNDPK2, TcNDPK 3 could be also associated to the cytoskeleton. To test this hypothesis, trypomastigote samples were analysed by immunofluorescence techniques using anti-TcNDPK3 antibodies. Interestingly, no signal was observed in the cytoskeleton, but fluorescence was located in multiple glycosome-like organelles. To further confirm these data, colocalisation analysis was performed using anti-hexokinase antibodies as glycosomal marker (Cazzulo 1992). As figure 3B shows, TcNDPK3 colocalises with hexokinase demonstrating that this NDPK isoform is present in glycosomes.

\section{DISCUSSION}

In this work we report for the first time the presence of a nucleoside diphosphate kinase in glycosomes, which is expressed only in the mammalian parasite stage trypomastigote. This could be explained because, in contrast to the insect stage, glycolytic enzymes are very abundant in bloodstream stages of mammalian trypanosomatids, which rely mainly on glycolysis and substrate-level phosphorylation for the generation of energy (Clayton and Michels 1996). In addition, the enzymatic content of glycosomes is rapidly changed during parasite stage differentiation (Michels et al. 2006). Although TcNDPK3 role in the glycosome is not obvious, it could be related to the nucleotide balance linked to the exchange across the membrane by specific nucleotide transporters. Nevertheless, if this is the actual TcNDPK3 function it may be exclusive for Trypanosoma cruzi as no NDPK3-like protein have been found in glycosomal proteomes in other trypanosomatid organisms.

An other singular feature of TcNDPK 3 is the apparent absence of known peroxisomal targeting signals (PTS). However, the subcellular localisation could be related with the quaternary structure of the protein, since many NDPKs have shown to form homohexamers. Using prediction programs, TcNDPK3 has low expected values for the con- 


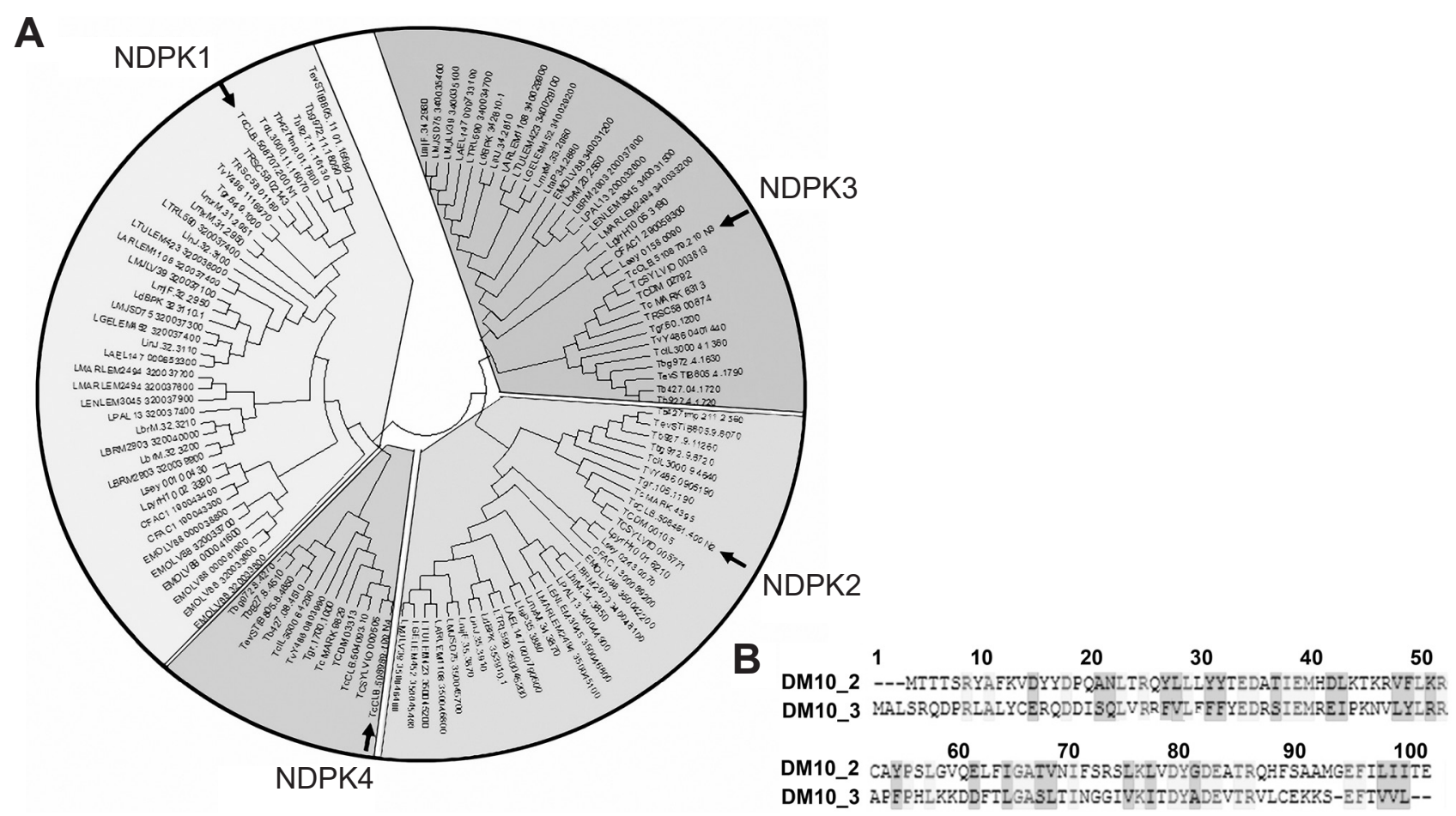

Fig. 2. Phylogenetic analysis of nucleoside diphosphate kinases (NDPKs) of trypanosomatids. A - orthologous genes of Trypanosoma cruzi nucleoside diphosphate kinase 1-Trypanosoma cruzi nucleoside diphosphate kinase 4 (TcNDPK1-TcNDPK4) were obtained from the available genomes of species of the genera Crithidia, Endotrypanum, Leishmania, Leptomonas and Trypanosoma. Using the obtained 118 genes a Maximum Likelihood unrooted phylogenetic tree was constructed with the MEGA software. The four clusters of the gene orthologs were separated by lines and NDPKs of T. cruzi were indicated (arrow); B - amino acid sequences of the DM10 domain corresponding to TcNDPK2 (DM10_2) and TcNDPK3 (DM10_3) were aligned and identical (light-gray coloured) and equivalent (dark-grey coloured) amino acids were indicated.
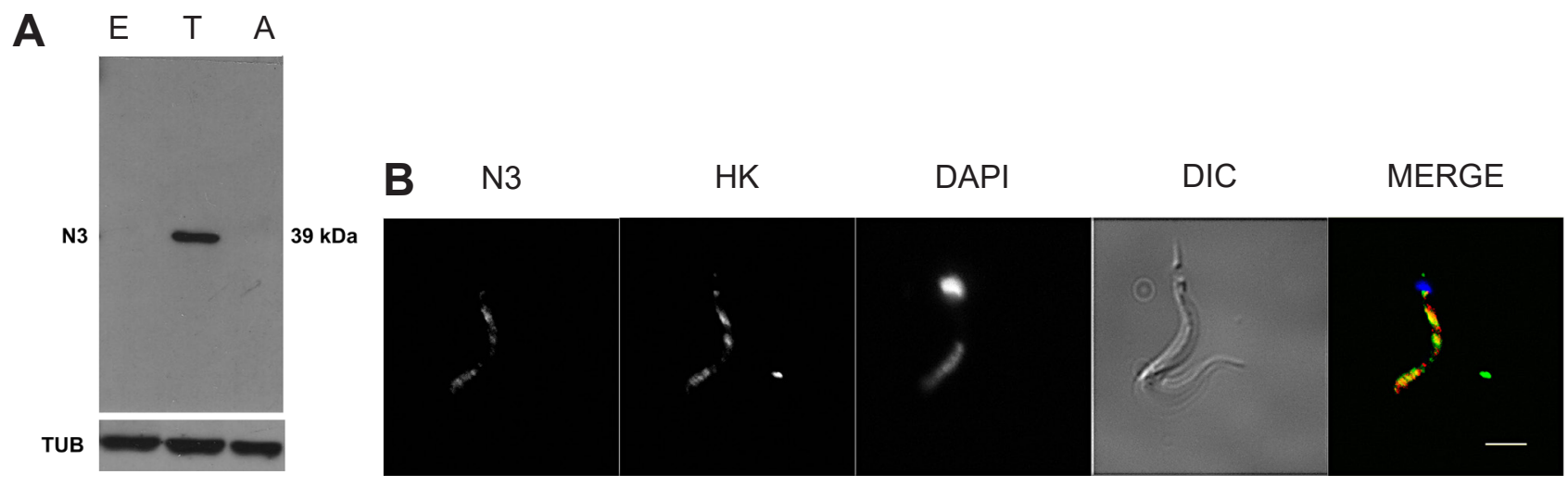

Fig. 3. Trypanosoma cruzi nucleoside diphosphate 3 (TcNDPK3) expression analysis and subcellular localisation. A - Western blots were performed using samples from the main stages of the life cycle of Trypanosoma cruzi, epimastigotes (E), trypomastigotes (T) and amastigotes (A). Upper panel was revealed using anti-TcNDPK3 antibodies (N3) and the lower panel using anti-tubulin antibodies as loading control (TUB). Molecular weights are also indicated; B - immunofluorescence microscopy was performed using samples of trypomastigotes and anti-TcNDPK3 antibodies (N3), anti-hexokinase antibodies (HK), DAPI staining and differential interference contrast (DIC). TcNDPK3 and hexokinase signals were merged to detect the colocalisation of both enzymes (MERGE).

sensus sequences PTS1, PTS2 and Pex19, suggesting other localisation signals which were not yet identified. The putative role of the DM10 domain in TcNDPK3 localisation could not be tested because the parasites that express this domain fused to a fluorescence marker or a truncated TcNDPK3 have a lethal phenotype (data not shown). Therefore, further studies are required to confirm the DM10 domain function in this protein. Since TcNDPK2 is associated with microtubules and it has the DM10 domain, TcNDPK3 could be located inside glycosomes or could be bound to the outside of the organelle in association with any specific structure. TcNDPK3 seems to be regulated at mRNA and protein levels since the mRNA quantified by RT-PCR was detected mainly in trypomastigote but also in samples of amastigotes, unlike the protein that was present exclusively in trypomastigotes.

The evolutionary importance of TcNDPK3 is reflected in its conservation among different kinetoplastid organisms 
as it is the second most represented isoform with 33 gene orthologs. Other phosphotransferase enzymes involved in nucleotide homeostasis have been previously identified in trypanosomatid glycosomes, for example the adenylate kinase isoforms 3 and $\mathrm{D}$ in T. cruzi and T. brucei, respectively (Ginger et al. 2005, Cámara et al. 2012) and the arginine kinase isoform 2 in T. brucei (Voncken et al. 2013). Despite conservation throughout the evolution of a wide variety of phosphotransferases in glycosomes, their function in this organelle still remains unknown.

In addition, two human TcNDPK3 gene homologs were identified, corresponding to the nucleoside diphosphate kinase 7 isoforms A and B (GeneBank IDs: NP_037462.1 and NP 932076.1, respectively). Both human isoforms were associated to the risk of venous thromboembolism risk; however, there is also no information about the function of these enzymes (Heit et al. 2012). Interestingly, the
NDPK3 from T. cruzi showed the lowest affinity for its substrate dTDP when compared with the NDPKs from other organisms, with a Km 3-5 times higher than the reported for the human NDPKs. These differences in kinetics may suggest dissimilar functionalities in each organism that could be exploited for drug design.

Considering that glycosomes are present only in kinetoplastid, the identification of new enzymes of this organelle constitutes an important step for the development of new therapies for trypanosomatid-associated diseases.

Acknowledgements. This work was supported by Consejo Nacional de Investigaciones Científicas y Técnicas (CONICET, PIP 2010-0685 and 2013-0664), Agencia Nacional de Promoción Científica y Tecnológica (FONCYT PICT 2012-0559 and 20150539). MMC, LAB and CAP are members of the career of scientific investigator, CONICET; CR and MS are research fellows from CONICET.

\section{REFERENCES}

Berriman M., Ghedin E., Hertz-Fowler C., Blandin G., Renauld H., Bartholomeu D.C., Lennard N.J., Caler E., Hamlin N.E., Haas B., Bohme U., Hannick L., Aslett M.A., Shallom J., Marcello L., Hou L., Wickstead B., Alsmark U.C., Arrowsmith C., Atkin R.J., Barron A.J., Bringaud F., Brooks K., Carrington M., Cherevach I., Chillingworth T.J., Churcher C., Clark L.N., Corton C.H., Cronin A., Davies R.M., Doggett J., Djikeng A., Feldblyum T., Field M.C., Fraser A., Goodhead I., Hance Z., Har per D., Harris B.R., Hauser H., Hostetler J., Ivens A., Jagels K., Johnson D., Johnson J., Jones K., Kerhornou A.X., Koo H., Larke N., Landfear S., LarKin C., Leech V., Line A., Lord A., Macleod A., Mooney P.J., Moule S., Martin D.M., Morgan G.W., Mungall K., Norbertczak H., Ormond D., Pai G., Peacock C.S., PeTerson J., Quail M.A., Rabbinowitsch E., Rajandream M.A., Reitter C., Salzberg S.L., Sanders M., Schobel S. Sharp S., Simmonds M., Simpson A.J., Tallon L., Turner C.M., Tait A., Tivey A.R., Van Aken S., Walker D., Wanless D., Wang S., White B., White O., Whitehead S., Woodward J., Wortman J., Adams M.D., Embley T.M., Gull K., Ullu E., Barry J.D., Fairlamb A.H., Opperdoes F., Barrell B.G., Donelson J.E., Hall N., Fraser C.M., Melville S.E., El-SAyed N.M. 2005: The genome of the African trypanosome Trypanosoma brucei. Science 309: 416-422.

Cámara M.M., Bouvier L.A., Miranda M.R., Pereira C.A 2012: Identification and validation of Trypanosoma cruzi's glycosomal adenylate kinase containing a peroxisomal targeting signal. Exp. Parasitol. 130: 408-411.

Camargo E.P. 1964: Growth and differentiation in Trypanosoma cruzi. Origin of metacyclic trypanosomes in liquid media. Rev. Inst. Med. Trop. São Paulo 6: 93-100.

CAzzulo J.J. 1992: Aerobic fermentation of glucose by trypanosomatids. FASEB J. 6: 3153-3161.

Clayton C.E., Michels P. 1996: Metabolic compartmentation in African trypanosomes. Parasitol. Today 12: 465-471.

Debrabant A., Nakhasi H. 2003: Programmed cell death in trypanosomatids: is it an altruistic mechanism for survival of the fittest? Kinetoplastid Biol. Dis. 2: 7.

Geiger A., Hirtz C., Becue T., Bellard E., Centeno D., Gargani D., Rossignol M., Cuny G., Peltier J.B. 2010: Exocytosis and protein secretion in Trypanosoma. BMC Microbiol. 10: 20.

Ginger M.L., Ngazoa E.S., Pereira C.A., Pullen T.J., KabiRi M., Becker K., Gull K., Steverding D. 2005: Intracellular positioning of isoforms explains an unusually large adenylate kinase gene family in the parasite Trypanosoma brucei. J. Biol. Chem. 280: 11781-11789.

Gomez Barroso J.A., Pereira H., Miranda M., Pereira C., Garratt R.C., Aguilar C.F. 2010: Protein preparation, crystallization and preliminary X-ray analysis of Trypanosoma cruzi nucleoside diphosphate kinase 1. Acta Crystallogr. Sect. F. Struct. Biol. Cryst. Commun. 66: 862-865.

Hammargren J., Salinas T., Marechal-Drouard L., KnorpP C. 2007: The pea mitochondrial nucleoside diphosphate kinase cleaves DNA and RNA. FEBS Lett. 581: 3507-3511.

Heit J.A., Armasu S.M., Asmann Y.W., Cunningham J.M., Matsumoto M.E., Petterson T.M., DE Andrade M. 2012 A genome-wide association study of venous thromboembolism identifies risk variants in chromosomes 1q24.2 and 9q. J. Thromb. Haemost. 10: 1521-1531.

Hunger-Glaser I., Hemphill A., Shalaby T., Hanni M., SeeBECK T. 2000: Nucleoside diphosphate kinase of Trypanosoma brucei. Gene 257: 251-257.

Kolli B.K., Kostal J., Zaborina O., Chakrabarty A.M., CHANG K.P. 2008: Leishmania-released nucleoside diphosphate kinase prevents ATP-mediated cytolysis of macrophages. Mol. Biochem. Parasitol. 158: 163-175.

Michels P.A., Bringaud F., Herman M., Hannaert V. 2006: Metabolic functions of glycosomes in trypanosomatids. Biochim. Biophys. Acta 1763: 1463-1477.

Miranda M.R., Cámara M.M., Bouvier L.A., Pereira C.A. 2011: TcNDPK2, a Trypanosoma cruzi microtubule-associated nucleoside diphosphate kinase. Mol. Biochem. Parasitol. 177: $152-155$.

Miranda M.R., Canepa G.E., Bouvier L.A., Pereira C.A. 2008a: Trypanosoma cruzi: multiple nucleoside diphosphate kinase isoforms in a single cell. Exp. Parasitol. 120: 103-107.

Miranda M.R., Canepa G.E., Bouvier L.A., Pereira C.A. 2008b: Trypanosoma cruzi nucleoside diphosphate kinase 1 ( TcNDPK1) has a broad nuclease activity. Parasitology 135: 1661-1666.

de Oliveira A.H., Ruiz J.C., Cruz A.K., Greene L.J., Rosa J.C., WARD R.J. 2006: Subproteomic analysis of soluble proteins of the microsomal fraction from two Leishmania species. Comp. Biochem. Physiol. Part D Genomics Proteomics 1: 300308.

Pereira C.A., Reigada C., Saye M., Digirolamo F.A., MiranDA M.R. 2014: Cytosolic Trypanosoma cruzi nucleoside diphosphate kinase generates large granules that depend on its quaternary structure. Exp. Parasitol. 142: 43-50. 
Postel E.H. 2003: Multiple biochemical activities of NM23/NDP kinase in gene regulation. J. Bioenerg. Biomembr. 35: 31-40.

Tamura K., Stecher G., Peterson D., Filipski A., Kumar S. 2013: MEGA6: Molecular Evolutionary Genetics Analysis version 6.0. Mol. Biol. Evol. 30: 2725-2729.

Ulloa R.M., Muschietti J.P., Veron M., Torres H.N., Tellez-InON M.T. 1995: Purification and characterization of a soluble nucleoside diphosphate kinase in Trypanosoma cruzi. Mol. Biochem. Parasitol. 70: 119-129.
Veron M., Tepper A., Hildebrandt M., Lascu I., Lacombe M.L., Janin J., Morera S., Cherfils J., Dumas C., Chiadmi M. 1994: Nucleoside diphosphate kinase: an old enzyme with new functions? Adv. Exp. Med. Biol. 370: 607-611.

Voncken F., Gao F., Wadforth C., Harley M., Colasante C. 2013: The phosphoarginine energy-buffering system of Trypanosoma brucei involves multiple arginine kinase isoforms with different subcellular locations. PLoS ONE 8: e65908.

Cite this article as: Cámara M.M., Bouvier L., Reigada C., Digirolamo F.A., Sayé M., Pereira C.A. 2017: A novel stage-specific glycosomal nucleoside diphosphate kinase from Trypanosoma cruzi. Folia Parasitol. 64: 006. 